\title{
Quantitative Forecasting of Risk for PTSD Using Ecological Factors: A Deep Learning Application
}

\author{
Nuriel S. Mor \\ Talpiot College of Education, Holon, Israel; Darca School, Bat Yam, Israel; and \\ Bnei Akiva School, Holon, Israel \\ Kathryn L. Dardeck \\ Walden University
}

\begin{abstract}
Forecasting the risk for mental disorders from early ecological information holds benefits for the individual and society. Computational models used in psychological research, however, are barriers to making such predictions at the individual level. Preexposure identification of future soldiers at risk for posttraumatic stress disorder (PTSD) and other individuals, such as humanitarian aid workers and journalists intending to be potentially exposed to traumatic events, is important for guiding decisions about exposure. The purpose of the present study was to evaluate a machine learning approach to identify individuals at risk for PTSD using readily collected ecological risk factors, which makes scanning a large population possible. An exhaustive literature review was conducted to identify multiple ecological risk factors for PTSD. A questionnaire assessing these factors was designed and distributed among residents of southern Israel who have been exposed to terror attacks; data were collected from 1,290 residents. A neural network classification algorithm was used to predict the likelihood of a PTSD diagnosis. Assessed by cross-validation, the prediction of PTSD diagnostic status yielded a mean area under receiver operating characteristics curve of $.91(F$ score $=.83)$. This study is a novel attempt to implement a neural network classification algorithm using ecological risk factors to predict potential risk for PTSD. Preexposure identification of future soldiers and other individuals at risk for PTSD from a large population of candidates is feasible using machine learning methods and readily collected ecological factors.
\end{abstract}

Keywords: PTSD, artificial neural network, machine learning, forecasting, ecological risk factors, southern Israel

\section{Introduction}

Certain people are at a higher risk to develop posttraumatic stress disorder (PTSD) following exposure to traumatic events than others (McKeever \& Huff, 2003). PTSD is a chronic mental disorder that causes impairments in important areas of functioning (American Psychiatric Association, 2013). The main characteristic symptoms of PTSD are recurrent distressing memories of the traumatic event, dissociative reactions, intense distress at exposure to cues that resemble the traumatic event, persistent avoidance symptoms, negative alteration in cognition and mood, and increased arousal and reactivity (American Psychiatric Association, 2013).

Please address queries to: Nuriel S. Mor, Talpiot College of Education, Holon, Israel; Darca School, Bat Yam, Israel; and Bnei Akiva School, Holon, Israel. Email: nuriel.mor@waldenu.edu 


\section{Etiology of PTSD}

Researchers have identified a large number of risk and protective factors for PTSD (Galatzer-Levy, Karstoft, Statnikov, \& Shalev, 2014; Gelkopf, Berger, Bleich, \& Silver, 2012; Mor \& Dardeck, 2017). The diathesis stress model of PTSD is a comprehensive etiological model that integrates up-to-date findings from both medical and psychological research (McKeever \& Huff, 2003). According to this model, three etiological pathways are associated with the disorder: residual stress, ecological diathesis, and biological diathesis (McKeever \& Huff, 2003). Residual stress comes from the severity of the trauma. Biological diathesis includes all the biological factors associated with the development of PTSD. Ecological diathesis includes personal, family, cultural, social, and environmental factors that may influence vulnerability for PTSD. Despite the fact that researchers have identified a large number of risk and protective factors for PTSD, the identification of individuals at risk for PTSD remains elusive (Galatzer-Levy et al., 2014).

The reason for the gap between scientific discoveries and the impact of these discoveries on clinical practice is the current statistical approach of psychological researches (Galatzer-Levy et al., 2014). Studies have typically reported statistical significance at the group level, but in practice, there is a need to make clinical decisions about individuals (Galatzer-Levy et al., 2014).

\section{Computational Challenge of Inferences at the Level of the Individual}

Inferences at the level of the individual require analytical methods that are designed for this purpose. The traditional methods of analysis based on general linear modeling (GLM) are restricted to testing specific hypotheses without an ability to generalize and give predictions for data that was not under study (Galatzer-Levy et al., 2014). GLM allows inferring only at the group level as to how much variance in the dependent variable can be explained by the independent variables and calculating the probability of rejecting a null hypothesis (Galatzer-Levy et al., 2014; Orrù, Pettersson-Yeo, Marquand, Sartori, \& Mechelli, 2012). These traditional statistical methods of analysis do not allow inference about individuals' previously unseen data. GLM is restricted to testing specific hypotheses based on the data under study, without generalization beyond this known data (Faraway, 2016; Galatzer-Levy et al., 2014).

Inferences present a computational challenge at the individual level. Three etiological pathways are associated with PTSD, and each etiological pathway contains numerous different risk factors (McKeever \& Huff, 2003). Therefore, on the level of the individual, development of PTSD reflects an interaction of many different risk factors (Galatzer-Levy et al., 2014; McKeever \& Huff, 2003).

\section{Advantages of Machine Learning in Forecasting of PTSD at the Level of the Individual}

Machine learning (ML) is a field of study concerned with construction of algorithms that can learn from the data and make predictions on previously unseen data without explicit programming (Goodfellow, Bengio, \& Courville, 2016). Relative to GLM, the approach of ML has two main advantages relevant to the problem of identifying individual people who are at risk for PTSD. First, ML methods can model complex relationships between input and output, which is essential in the current problem of predicting the development of PTSD. A complex interaction between numerous risk factors with complex conditional dependencies determines levels of vulnerability for PTSD (Orrù et al., 2012), and ML factors these all in. Second, ML methods allow inferring at the level of the individual and allow categorization of an individual's previously unseen data, therefore yielding results with practical, clinical application (Orrù et al., 2012). 
The use of a ML approach to predict an outcome is increasingly used in developing individualized medicine and is known as forecasting (Galatzer-Levy et al., 2014). Numerous ML studies using biomarkers for neurological and psychological disorders such as Alzheimer's disease, schizophrenia, major depression, bipolar disorder, Huntington's disease, Parkinson's disease, autistic spectrum disorder, and PTSD have shown initial promising results in identifying them at the individual level (Gong et al., 2014; Orrù et al., 2012). The researchers of these studies used biomarkers and neuroimaging when the present study was based on ecological risk factors. A main weakness of approaches that use biomarkers obtained by neuroimaging is that neuroimaging is not often used in psychology because of the financial costs involved in such procedures (Galatzer-Levy et al., 2014).

\section{Previous Researches Applied Machine Learning to Predict PTSD}

A gap in the current literature exists regarding a lack of attempts at applying ML algorithms on ecological risk factors to predict PTSD (Galatzer-Levy, et al., 2014), despite diathesis being an etiological pathway that is associated with PTSD (McKeever \& Huff, 2003). Three studies that did apply ML algorithms on descriptive information are those of Galatzer-Levy et al. (2014); Karstoft, Statnikov, Andersen, Madsen, and Galatzer-Levy (2015); and Rosellini, Dussaillant, Zubizarreta, Kessler, and Rose (2018).

Galatzer-Levy et al. (2014) successfully used a ML approach to predict PTSD from early trauma responses. They collected various data such as demographics, blood pressure, pulse, pain level, and event type from 957 participants from Israel within 2 weeks of a traumatic event and applied a ML algorithm to predict PTSD diagnostic status more than a year following the traumatic event. Karstoft et al. (2015) successfully implemented ML algorithms using descriptive data such as age, gender, children (yes/no), parental divorce (yes/no), education level, smoking (yes/no), alcohol intake, and so on to identify Danish soldiers at risk for PTSD. Recently, Rosellini et al. (2018) successfully used ML methods to predict PTSD following earthquakes based on 67 risk factors that could be assessed within 1 week of earthquake occurrence. Rosellini and colleagues operationalized many risk factors such as sex, age, property damage, job loss, type of living area, and so forth. The present study was focused on PTSD from terror attacks, and a comprehensive list of ecological risk factors for PTSD from terror attacks was used.

\section{This Study's Approach}

The purpose of the current study was to evaluate the ability of a ML algorithm called artificial neural network to identify individuals at risk for PTSD using easily collected ecological risk factors for PTSD among residents of southern Israel. Recently, neural networks have become state of the art, modeling very complicated relationships between inputs and outputs (Srivastava, Hinton, Krizhevsky, Sutskever, \& Salakhutdinov, 2014).

Residents of southern Israel have been exposed to terror attacks since 2000 (Gelkopf et al., 2012; Stein et al., 2013). Using ML and ecological factors that can be easily collected by a questionnaire, as opposed to biological and neuroimaging data, helps to scan a very large population of people and identify those at risk for PTSD. Such a system holds great benefit because it helps to identify individuals at risk for PTSD from a very large population of people. Individual soldier candidates in Israel and elsewhere who are at risk for developing PTSD can therefore be identified from a very large population of candidates. Early preexposure identification of individuals at risk for PTSD may be very helpful for those intending to be exposed to stressors and traumatic events, such as soldiers who cannot afford to decompensate in a life and death situation. This could not only include soldiers, 
but also humanitarian aid workers, journalists, and medical personnel in war-torn and disaster areas.

\section{Method}

\section{Sample}

The target population for this study included residents of southern Israel who have been exposed to terror attacks since 2000 (Gelkopf et al., 2012; Mor \& Dardeck, 2017; Stein et al., 2013). Participants were recruited voluntarily in southern Israel. About 2,500 short paper questionnaires and an online form embedded in a website designed for this study were distributed. A total of 1,290 participants submitted their responses to these questionnaires.

\section{Measures}

An exhaustive literature review was conducted with the aim of identifying ecological risk factors for PTSD. The main categories of ecological risk factors that were identified were socioeconomic status (Luz, Berger, Figueira, Coutinho, \& Mendlowicz, 2015), age, education, gender (Xue et al., 2015), religiosity (Bentley, Ahmad, \& Thoburn, 2014), social support (Stanley et al., 2018), economic loss following the traumatic event (Gelkopf et al., 2012), physical activity (Rosenbaum et al., 2015), substance abuse (Bowen, De Boer, \& Bergman, 2017), family stressors (Vaughn-Coaxum, Smith, Iverson, \& Vogt, 2015), sense of belonging (Itzhaky, Weiss-Dagan, \& Taubman-Ben-Ari, 2018), level of exposure (Xue et al., 2015), intensity of trauma, and history of trauma (McKeever \& Huff, 2003; Stein et al., 2013; Xue et al., 2015). A short questionnaire of 37 questions measuring these factors was designed for the purpose of this study.

\section{Outcome Measure}

The outcome measure of this study is a dichotomized variable of no PTSD symptomatology versus PTSD symptomatology, which was measured by the last question of the questionnaire. The last question asked explicitly whether the participant had received a diagnosis of PTSD. Three hundred fifty-four (27.4\%) of the 1,290 study participants answered "yes" to this question.

\section{Modeling Approach}

\section{General Description of Modeling Approach}

The data contained ecological factors for PTSD and PTSD diagnostic status for each participant as assessed by the last question, which explicitly asked whether the participant had received a diagnosis of PTSD. In the present study, the ecological factors of each participant (socioeconomic status, education, age, gender, religiosity, social support, economic loss following the traumatic event, physical activity, substance abuse, family stressors, sense of belonging, level of exposure, intensity of trauma, and history of trauma) are the input to the neural network, and PTSD diagnostic status is the output. The artificial neural network, in the first stage, learns the relationship between the ecological factors and PTSD diagnostic status over the collected data of all participants. The neural network is intended in the second stage to provide a likelihood of a PTSD diagnosis for a new set of ecological factors of a new individual by generalizing the learning of the first stage. 


\section{Neural Networks for Machine Learning}

Neural networks for ML contain multiple hidden nonlinear layers, and this makes them expressive models with computational capacity that can learn complicated relationships between their inputs and outputs (Srivastava et al., 2014). A deep neural network is suitable to model a complicated relationship between inputs and outputs when complex conditional dependencies between variables exist, as in the current study problem of identifying those that at risk for PTSD given their ecological risk factors for PTSD (Galatzer-Levy et al., 2014)

\section{Cross-Validation}

To examine classification accuracy and generalizability of the model, a 10-fold cross-validation was applied, resulting in a total of 10 model building and testing runs. In 10 -fold cross-validation, the data is randomly partitioned into 10 equal sized subsets. A single subset is retained as the validation data for testing the model, and the remaining nine subsets are used as training data (Hornung, Bernau, Truntzer, Stadler, \& Boulesteix, 2014). The mean of performance metrics across the 10 cross-validations was used to estimate classification accuracy and generalizability of the model.

\section{Accuracy Metrics}

Two accuracy metrics were used to estimate predictive accuracy of the constructed model: area under the curve (AUC) and $F$ score. All the metric values represent the mean of each metric across 10 -fold cross-validation. AUC is defined by the area between the receiver operating characteristic curve and the $x$-axis and is given by the definite integral below. The receiver operating characteristic curve is created by plotting the true positive rate against the false positive rate at different threshold settings (Majnik \& Bosnić, 2013). AUC provides an unbiased measure of accuracy and can be directly compared to AUC obtained by a different system (Karstoft et al., 2015). F score is a weighted average of the precision and recall and a balanced performance measure (Hand \& Christen, 2018).

\section{Experiments and Results}

\section{Descriptive Statistics}

Of those who submitted the form, 705 (54.6\%) were males and 585 (45.4\%) were females. Table 1 summarizes additional demographic characteristics of the study sample.

Consistent with previous researchers (Gelkopf et al., 2012; Mor \& Dardeck, 2017; Stein et al., 2013) who examined prevalence of PTSD symptoms among Sderot, Israel, residents, $354(27.4 \%)$ of the 1,290 study participants reported they had been diagnosed with PTSD.

\section{Main Analyses}

\section{Designing the Network's Architecture}

Figure 1 presents the trial-and-error process of assessing the performance of each architecture while considering the shrinking structure principle (Zhang, Bao, Zhou, Jiang, \& Dai, 2014). Architecture refers here to the number of hidden layers in the neural network and number of units in each layer. The architecture with the highest value of accuracy metric that was found in cross-validation was a neural network with three hidden layers. These findings that the increased number of hidden layers increased the performance on the validation set up to a certain point, and that a further increase in hidden layers led to a decreased performance, are consistent with other researchers' findings (Erhan, Manzagol, Bengio, Bengio, \& Vincent, 2009). 
Table 1. Demographic Characteristics of Study Sample $(N=1,290)$

\begin{tabular}{lcc}
\hline Demographic & $n$ & $\%$ \\
\hline Age & 388 & 30.07 \\
$18-27$ & 340 & 26.3 \\
$28-37$ & 138 & 10.6 \\
$38-47$ & 197 & 15.3 \\
$48-57$ & 141 & 10.9 \\
$58-67$ & 53 & 4.1 \\
$68-77$ & 33 & 2.5 \\
$78-87$ & & \\
Education & 116 & 9 \\
Some elementary school & 160 & 12.4 \\
Graduate elementary & & \\
school & 592 & 45.9 \\
Some high school & 246 & 19 \\
High school graduate & 176 & 13.7 \\
Above high school & & \\
\hline
\end{tabular}

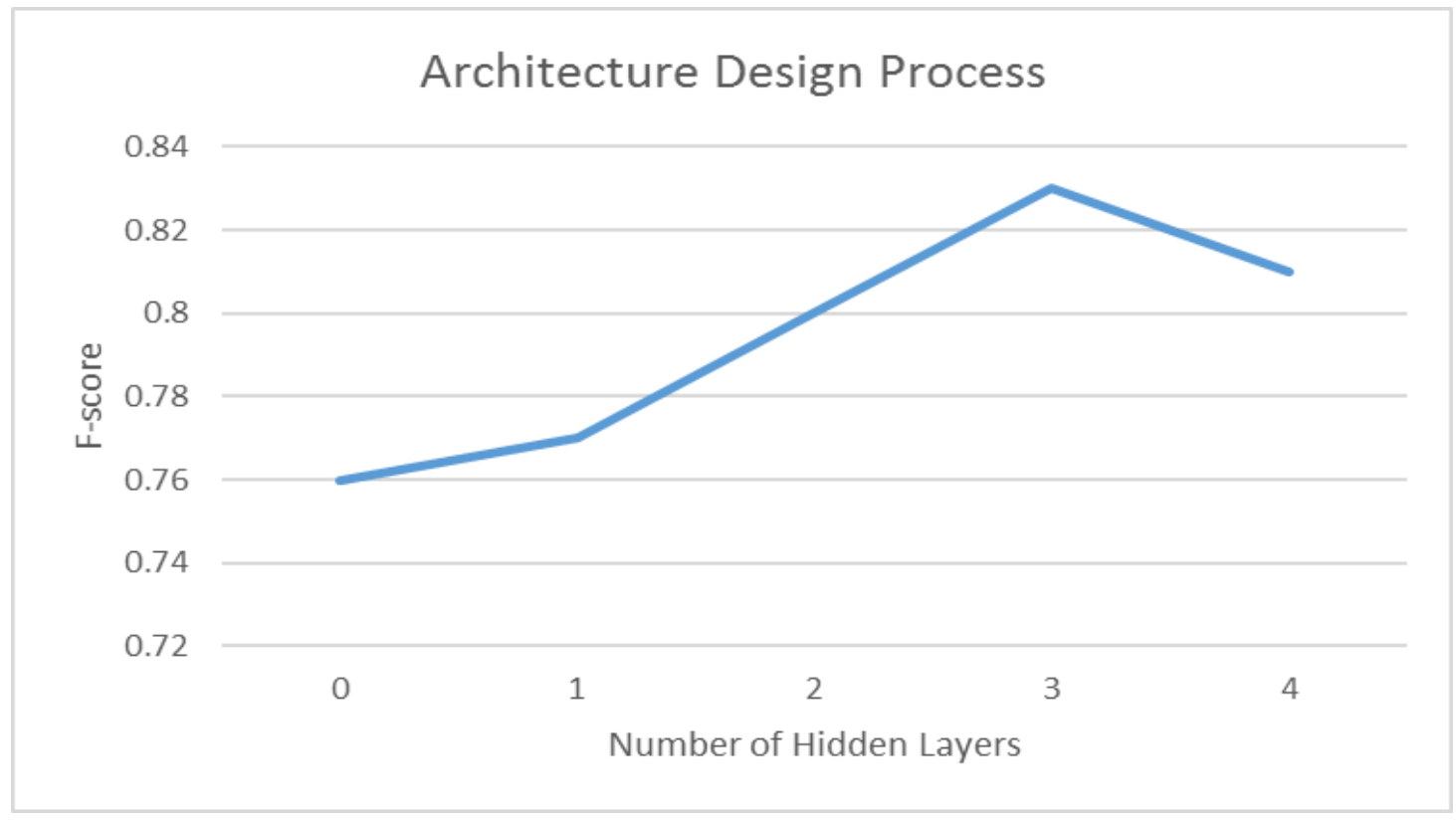

Figure 1. Trial-and-Error Process of Finding the Best Network Architecture. 


\section{Predicting PTSD Status}

The prediction of PTSD diagnostic status with the best architecture that was found yielded AUC = $.91(95 \%$ confidence interval $[0.89,0.93]), F$ score $=.83$ (95\% confidence interval $[0.82,0.84])$.

\section{Discussion}

This study evaluated the implementation of an artificial neural network algorithm to forecast PTSD using ecological risk factors for PTSD that can be easily collected. The ecological factors have been selected through an exhaustive literature review that identified many ecological factors that researchers (Bentley et al., 2014; Bowen et al., 2017; Gelkopf et al., 2012; Itzhaky et al., 2018; Luz et al., 2015; McKeever \& Huff, 2003; Rosenbaum et al., 2015; Stanley et al., 2018; Stein et al., 2013; Vaughn-Coaxum et al., 2015; Xue et al., 2015) found to influence PTSD risk.

This was the first study that implemented a ML method to forecast individuals at risk for PTSD using a comprehensive list of ecological risk factors. The ecological risk factors that were used and assessed in this study were age, gender, marital status, number of children, number of siblings, parents' marital status, monthly salary, financial hardship, economic worry, level of education, employment status, religiosity, social support, perceived government support, confidence in the Israeli Defense Force, family stressors, relatives who have been diagnosed with PTSD, economic loss following the traumatic event, number of days of exercise per week, alcohol intake, sense of belonging, sense of safety, sense of danger, exposure, and history of trauma.

The present study's model yielded an AUC of 0.91. AUC >.80 indicates a good prediction (GalatzerLevy et al., 2014). The finding that easily collected data provides efficient forecasting of people at high risk for PTSD is very promising.

A paucity of data exists regarding implementation of ML methods on readily collected descriptive information and ecological risk factors that do not involve technology to predict PTSD (Galatzer-Levy et al., 2014). Karstoft et al. (2015) applied ML methods to identify soldiers at risk for PTSD. The data on which Karstoft and colleagues implemented the ML algorithm were various descriptive and demographics features, such as age, gender, children (yes/no), parental divorce (yes/no), education level, smoking (yes/no), and alcohol intake. The accuracy obtained in this study is higher than the AUCs of previous studies that used descriptive information (Galatzer-Levy et al., 2014; Karstoft et al., 2015).

The weakness of previous studies (Galatzer-Levy et al., 2014; Karstoft et al., 2015) whose authors did use descriptive information and ML methods to predict PTSD is that the descriptive information did not include a comprehensive list of known ecological risk factors. Therefore, their ability to design an algorithm to predict PTSD given only a partial list of ecological risk factors for PTSD was inherently restricted.

The main strength of this study is derived from selecting appropriate ecological risk factors combined with use of a deep neural network. Studies in the psychological area have used GLM, which cannot provide predictions at the individual level (Galatzer-Levy et al., 2014; Orrù et al., 2012). Further, GLM is not suitable when conditional dependencies between variables exist. Therefore, GLM is not a suitable statistical approach for modeling the relationship between a PTSDmulticlausal etiology and the outcome measure (Galatzer-Levy et al., 2014; Orrù et al., 2012). 


\section{Applicability of This Study}

Using a ML approach and readily collected ecological risk factors to predict risk for PTSD holds significant benefits for individuals and society. A system based on a ML algorithm and readily collected ecological factors-as designed for this study - helps scan a very large population of people and identify those individuals who are at risk for PTSD.

The procedure of this study, based on a ML approach and ecological risk factors (as opposed to biological/neuroimaging data), can be readily translated to a prediction tool by implementing this procedure as a user-friendly app or website that scans a large population and receives predictions for each individual. To demonstrate this advantage, a novel Android app (which can be downloaded from https://drive.google.com/file/d/0B29CqHUg4JA6SHdINXpXWmF2M1k/view) and website (http://medical-predictions.appspot.com/) were developed based on this study's predictive model. The user-friendly interface allows users to scan a large population of people and easily receive predictions of risk for PTSD for each individual of that population (Figure 2). In addition, the system supports updating the model, expanding the data store and further developing the neural network (Figure 3). Figure 4 presents the general architecture of the system. The cost of this procedure is minimal and requires only answering an online questionnaire. Scanning a very large population of people at such a speed cannot be carried out by an in-depth interview. This kind of implementation can be very beneficial for big organizations like the army. Such a system as described in this study is intended to be a technological tool that helps identify people who are at risk of developing PTSD and is not intended to replace clinical interviews and treatment.

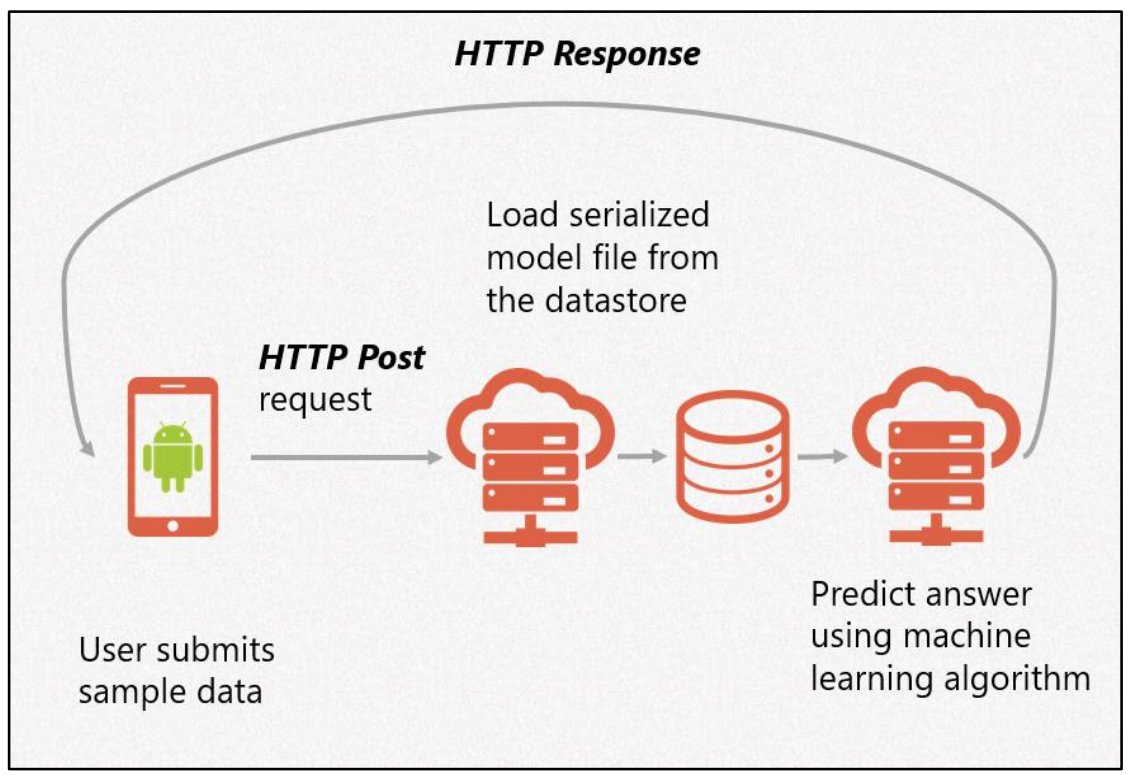

Figure 2. User Receives a Prediction. 


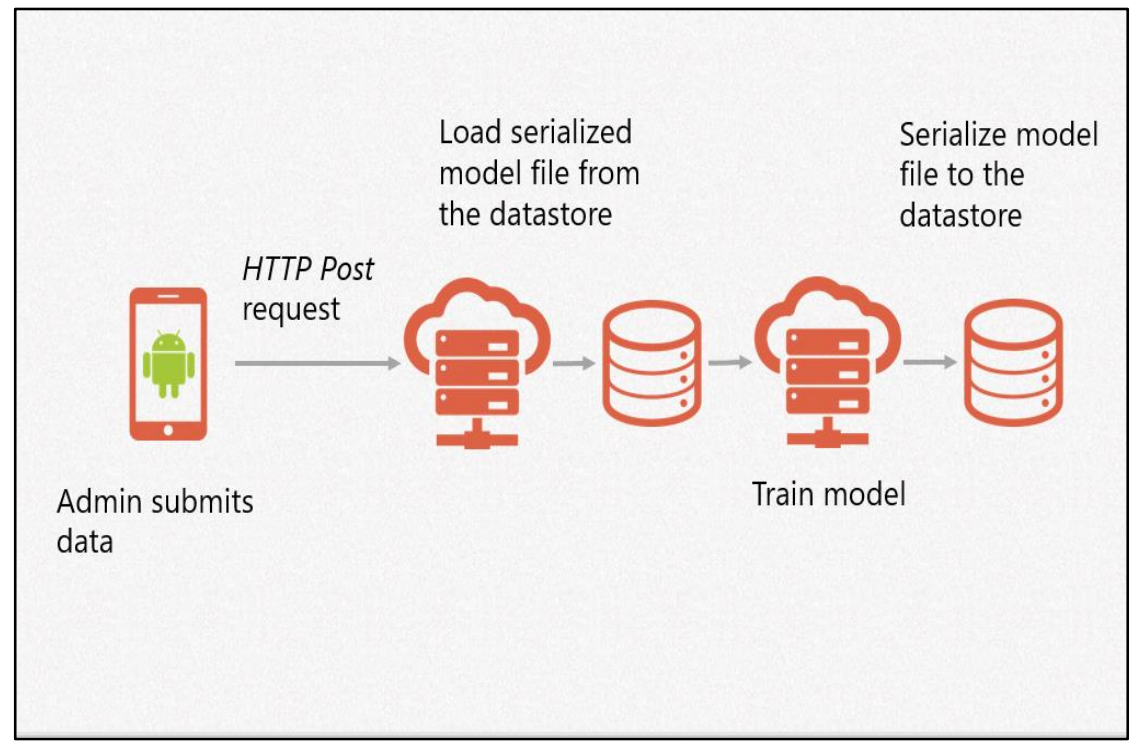

Figure 3. Administrator (Admin) Updates the Model.

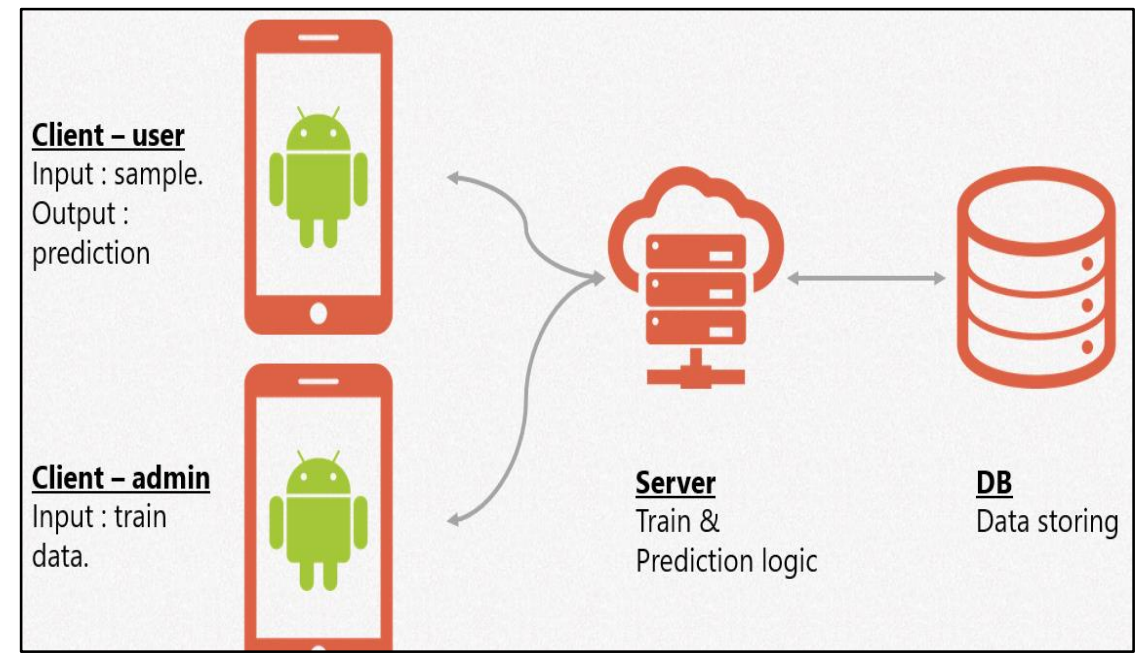

Figure 4. The General Architecture of the System. $D B=$ database. 
The present study demonstrated the inherent potential in ML techniques to identify (at the individual level) the risk for developing PTSD using ecological risk factors. The findings of this study suggest practical implications in identifying individuals at risk for PTSD. Early preexposure identification of individuals at risk for PTSD by readily collected ecological factors might be a helpful tool to identify people at risk who intend to be exposed to stressors and traumatic events. Naturally, future soldiers are the most suitable population for such a system given the fact that it can help to identify future soldiers at risk for PTSD from a very large population of candidates. The type of system that was built in this study may also help other populations, such as humanitarian aid workers, journalists, and medical personnel in war-torn and disaster areas who may be exposed to traumatic stressors, by guiding their decisions about exposure.

\section{Limitations}

This study is an initial and novel attempt to implement ML algorithms using comprehensive ecological risk factors to predict individuals at risk for PTSD. The following paragraphs describe the main limitations of this study, which future studies should address.

\section{Type of Traumatic Event}

This study was conducted on residents of southern Israel. The type of traumatic events experienced was chronic terror attacks. Therefore, the generalizability of this study is limited to PTSD caused by chronic terror attacks.

\section{Participants and Population}

As this study was conducted on residents of southern Israel, the results of this initial study cannot be generalized beyond residents of this region. Moreover, the participants included a convenience sample rather than a representative sample. Therefore, the generalizability of the results even to southern Israel residents is limited (Campbell \& Stanley, 1963). In addition, participation was voluntary. Therefore, those who agreed to volunteer for the study may be different from those who refused (Wiersma, 2013). Thus, the sample may be unrepresentative of the entire population of southern Israel and, as a result, the external validity of the results may be restricted (Wiersma, 2013).

\section{Outcome Measure}

The outcome measure of this study was a dichotomized variable of no PTSD versus PTSD, which was measured by the last question of the questionnaire. This study was an initial attempt to predict individuals at risk for PTSD using ecological risk factors. A more appropriate outcome measure such as the PTSD Checklist-Civilian Version (Weathers, Litz, Herman, Huska, \& Keane, 1994) should be applied in future studies, which might require extra time and effort in the information collection process.

\section{Recommendations for Future Work}

A first recommendation derived from the study's limitations is to collect data from many different populations to increase the external validity and applicability of the predictive model. A second recommendation is to use continuous variables and to code them into continuous rather than dichotomous variables. In this study, all variables were coded into dichotomous variables, which caused information loss. Continuous variables can contain more information about the level of each risk factor for each individual, not only represent presence or absence of each risk factor. In addition, the study outcome measure was also a dichotomized variable (PTSD versus no PTSD), as were all 
variables in this study. A more appropriate outcome measure for PTSD diagnostic status such as the PTSD Checklist-Civilian Version (Weathers et al., 1994) should be applied in future studies.

\section{Summary and Conclusion}

The present study demonstrated the feasibility of predicting PTSD using ecological risk factors. Using a ML approach and readily collected ecological risk factors makes scanning a large population possible to identify individuals at high risk for PTSD. Study findings can be a foundation for the future development of a helping tool to scan a large population and identify those at risk for PTSD from terror attacks. It also is suitable to identify future soldiers at risk of PTSD from a very large population of candidates. The model designed in this study is not an optimal solution because the study was conducted in southern Israel, and its external validity is restricted to this population. This study's findings, however, can be an inspiration for the future development of such an algorithm on many different populations across the world. In addition, the study's findings suggest, in accordance with the diathesis stress model of PTSD, that researchers need to incorporate ecological risk factors in addition to biological factors in predictive models of PTSD to offer a more optimal solution to predicting PTSD.

\section{References}

American Psychiatric Association. (2013). Diagnostic and statistical manual of mental disorders (5th ed.). Arlington, VA: Author.

Bentley, J., Ahmad, Z., \& Thoburn, J. (2014). Religiosity and posttraumatic stress in a sample of East African refugees. Mental Health, Religion and Culture, 17, 185-195.

Bowen, S., De Boer, D., \& Bergman, A. L. (2017). The role of mindfulness as approach-based coping in the PTSD-substance abuse cycle. Addictive Behaviors, 64, 212-226.

Campbell, D. T., \& Stanley, J. C. (1963). Experimental and quasi experimental designs for research. Boston, MA: Houghton Mifflin.

Erhan, D., Manzagol, P. A., Bengio, Y., Bengio, S., \& Vincent, P. (2009). The difficulty of training deep architectures and the effect of unsupervised pre-training. In D. van Dyk \& M. Welling (Eds.), Proceedings of the 12th International Conference on Artificial Intelligence and Statistics (pp. 153-160), Clearwater Beach, FL: Proceedings of Machine Learning Research. Retrieved from http://proceedings.mlr.press/v5/erhan09a/erhan09a.pdf

Faraway, J. J. (2016). Extending the linear model with R: Generalized linear, mixed effects and nonparametric regression models (2nd ed.). Boca Raton, FL: CRC Press.

Galatzer-Levy, I. R., Karstoft, K. I., Statnikov, A., \& Shalev, A. Y. (2014). Quantitative forecasting of PTSD from early trauma responses: A machine learning application. Journal of Psychiatric Research, 59, 68-76.

Gelkopf, M., Berger, R., Bleich, A., \& Silver, R. C. (2012). Protective factors and predictors of vulnerability to chronic stress: A comparative study of 4 communities after 7 years of continuous rocket fire. Social Science and Medicine,74, 757-766.

Gong, Q., Li, L., Tognin, S., Wu, Q., Pettersson-Yeo, W., Lui, S., \& Mechelli, A. (2014). Using structural neuroanatomy to identify trauma survivors with and without post-traumatic stress disorder at the individual level. Psychological Medicine, 44, 195-203.

Goodfellow, I., Bengio, Y., \& Courville, A., (2016). Deep learning. Cambridge, MA: MIT Press. 
Hand, D., \& Christen, P. (2018). A note on using the $F$-measure for evaluating record linkage algorithms. Statistics and Computing, 28, 539-547.

Hornung, R., Bernau, C., Truntzer, C., Stadler, T., \& Boulesteix, A. (2014). Full versus incomplete cross-validation: Measuring the impact of imperfect separation between training and test sets in prediction error estimation (Technical Report No. 159). Department of Statistics, Ludwig Maximilian University of Munich, Munich, Germany. Retrieved from https://epub.ub.unimuenchen.de/20682/1/TR.pdf

Itzhaky, H., Weiss-Dagan, S., \& Taubman-Ben-Ari, O. (2018). Internal and community recourses' contribution to level of posttraumatic symptoms: The case of tourists after the earthquake in Nepal, 2015. Psychological Trauma: Theory, Research, Practice, and Policy, 10, 239-245.

Karstoft, K. I., Statnikov, A., Andersen, S. B., Madsen, T., \& Galatzer-Levy, I. R. (2015). Early identification of posttraumatic stress following military deployment: Application of machine learning methods to a prospective study of Danish soldiers. Journal of affective disorders, 184, 170-175.

Luz, M., Berger, W., Figueira, I., Coutinho, E. S. F., \& Mendlowicz, M. (2015). A calculated risk: Systematic review and metaanalysis of the conditional risk for posttraumatic stress disorder (PTSD) in the Brazilian general population. European Psychiatry, 30, 1546a-1546d.

Majnik, M., \& Bosnić, Z. (2013). ROC analysis of classifiers in machine learning: A survey. Intelligent Data Analysis, 17, 531-558.

McKeever, V. M., \& Huff, M. E. (2003). A diathesis-stress model of posttraumatic stress disorder: Ecological, biological, and residual stress pathways. Review of General Psychology, 7, 237250.

Mor, N. S., \& Dardeck, K. L., (2017). Mitigation of posttraumatic stress symptoms from chronic terror attacks on southern Israel. Journal of Social, Behavioral, and Health Sciences, 11, $47-64$.

Orrù, G., Pettersson-Yeo, W., Marquand, A. F., Sartori, G., \& Mechelli, A. (2012). Using support vector machine to identify imaging biomarkers of neurological and psychiatric disease: A critical review. Neuroscience and Biobehavioral Reviews, 36, 1140-1152.

Rosellini, A. J., Dussaillant, F., Zubizarreta, J. R., Kessler, R. C., \& Rose, S. (2018). Predicting posttraumatic stress disorder following a natural disaster. Journal of Psychiatric Research, $96,15-22$.

Rosenbaum, S., Vancampfort, D., Steel, Z., Newby, J., Ward, P. B., \& Stubbs, B. (2015). Physical activity in the treatment of post-traumatic stress disorder: A systematic review and metaanalysis. Psychiatry Research, 230, 130-136.

Srivastava, N., Hinton, G., Krizhevsky, A., Sutskever, I., \& Salakhutdinov, R. (2014). Dropout: A simple way to prevent neural networks from overfitting. The Journal of Machine Learning Research, 15, 1929-1958.

Stanley, I. H., Hom, M. A., Chu, C., Dougherty, S. P., Gallyer, A. J., Spencer-Thomas, S., ... SachsEricsson, N. J. (2018). Perceptions of belongingness and social support attenuate PTSD symptom severity among firefighters: A multistudy investigation. Psychological Services. Advance online publication. 
Stein, N. R., Schorr, Y., Krantz, L., Dickstein, B. D., Solomon, Z., Horesh, D., \& Litz, B. T. (2013). The differential impact of terrorism on two Israeli communities. American Journal of Orthopsychiatry, 83, 528-535.

Vaughn-Coaxum, R., Smith, B. N., Iverson, K. M., \& Vogt, D. (2015). Family stressors and postdeployment mental health in single versus partnered parents deployed in support of the wars in Afghanistan and Iraq. Psychological Services, 12, 241-249.

Weathers, F. W., Litz, B. T., Herman, D., Huska, J., \& Keane, T. (1994). The PTSD ChecklistCivilian version $(P C L-C)$. Boston, MA: National Center for PTSD.

Wiersma, W. (2013). The validity of surveys: Online and offline. Retrieved from http://wybowiersma.net/pub/essays/Wiersma,Wybo,The_validity_of_surveys_online_and_offli ne.pdf

Xue, C., Ge, Y., Tang, B., Liu, Y., Kang, P., Wang, M., \& Zhang, L. (2015). A meta-analysis of risk factors for combat-related PTSD among military personnel and veterans. PloS One, 10, e0120270.

Zhang, S., Bao, Y., Zhou, P., Jiang, H., \& Dai, L. (2014). Improving deep neural networks for LVCSR using dropout and shrinking structure. In IEEE International Conference of Acoustics, Speech, and Signal Processing (pp. 6849-6853). Florence, Italy: IEEE.

The Journal of Social, Behavioral, and Health Sciences is an open-access, peer-reviewed, online interdisciplinary journal focusing on research findings that address contemporary national and international issues. Its objectives are to (a) encourage dialogue between scholars and practitioners in the social, behavioral, and health sciences that fosters the integration of research with practice; (b) promote innovative models of interdisciplinary collaboration among the social, behavioral, and health sciences that address complex social problems; and (c) inform the relationship between practice and research in the social, behavioral, and health sciences.

Walden University Publishing: http://www.publishing.waldenu.edu 